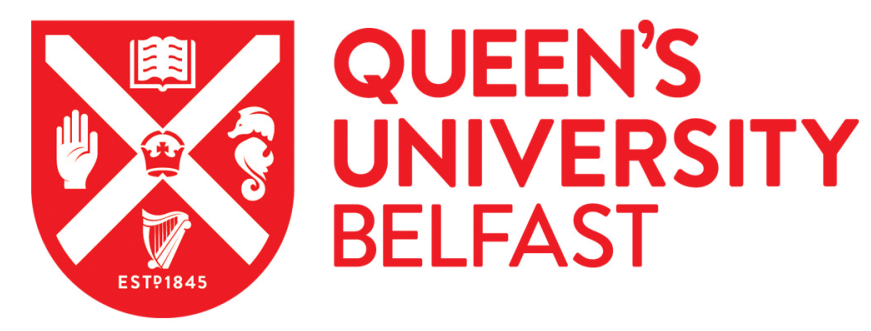

\title{
Perceptual Modeling of Tinnitus Pitch and Loudness
}

Gault, R., McGinnity, T. M., \& Coleman, S. (2020). Perceptual Modeling of Tinnitus Pitch and Loudness. IEEE Transactions on Cognitive and Developmental Systems , 12(2), 332-343. https://doi.org/10.1109/TCDS.2020.2964841

\section{Published in:}

IEEE Transactions on Cognitive and Developmental Systems

\section{Document Version:}

Peer reviewed version

\section{Queen's University Belfast - Research Portal:}

Link to publication record in Queen's University Belfast Research Portal

\section{Publisher rights}

(c) 2020 IEEE.

This work is made available online in accordance with the publisher's policies. Please refer to any applicable terms of use of the publisher.

\section{General rights}

Copyright for the publications made accessible via the Queen's University Belfast Research Portal is retained by the author(s) and / or other copyright owners and it is a condition of accessing these publications that users recognise and abide by the legal requirements associated with these rights.

Take down policy

The Research Portal is Queen's institutional repository that provides access to Queen's research output. Every effort has been made to ensure that content in the Research Portal does not infringe any person's rights, or applicable UK laws. If you discover content in the Research Portal that you believe breaches copyright or violates any law, please contact openaccess@qub.ac.uk. 


\title{
Perceptual modelling of tinnitus pitch and loudness
}

\author{
Richard Gault, Thomas Martin McGinnity, Senior Member, IEEE, and Sonya Coleman, Member, IEEE
}

\begin{abstract}
Tinnitus is the phantom perception of sound, experienced by $10-15 \%$ of the global population. Computational models have been used to investigate the mechanisms underlying the generation of tinnitus-related activity. However, existing computational models have rarely benchmarked the modelled perception of a phantom sound against recorded data relating to a person's perception of tinnitus characteristics; such as pitch or loudness. This paper details the development of two perceptual models of tinnitus. The models are validated using empirical data from people with tinnitus and the models' performance is compared with existing perceptual models of tinnitus pitch. The first model extends existing perceptual models of tinnitus, while the second model utilises an entirely novel approach to modelling tinnitus perception using a Linear Mixed Effects (LME) model. The LME model is also used to model the perceived loudness of the phantom sound which has not been considered in previous models. The LME model creates an accurate model of tinnitus pitch and loudness and shows that both tinnitus-related activity and individual perception of sound are factors in the perception of the phantom sound that characterizes tinnitus.
\end{abstract}

Index Terms-Tinnitus, perceptual model, linear mixed effects model, tinnitus pitch, tinnitus loudness.

\section{INTRODUCTION}

$\mathbf{S}$ UBJECTIVE tinnitus is the phantom perception of a sound heard in or around the head in the absence of an identifiable source and is experienced by $10-15 \%$ of the population [1]. Objective tinnitus occurs when the tinnitus sound is caused by a physical source. Furthermore, tinnitus can be described as pulsatile if the perceived sound appears as a pulsing sound synchronized to a person's heartbeat [2]; or otherwise it is described as non-pulsatile tinnitus. In this work we are only concerned with subjective non-pulsatile tinnitus which is the most common form of the phenomenon.

Tinnitus has been shown to be associated with observable differences in auditory and non-auditory areas of the central nervous system compared with healthy counterparts [3], [4], [5]. It is thought that most tinnitus cases stem from peripheral noise-induced hearing loss creating a reduction in auditory input [6] with approximately $90 \%$ of people with tinnitus have apparent hearing loss [7]. It is thought that the remaining people with tinnitus have hidden hearing loss [8], [9], [10], [11]. The loss of auditory input in regions of hearing loss causes adaptation within the auditory system leading to increased spontaneous neuronal activity, or hyperactivity, in the

$\mathrm{R}$ Gault is a member of the School of Electronics, Electrical Engineering and Computer Science, Queen's University, Belfast, U.K..E-mail: richard.gault@qub.ac.uk

TM. McGinnity is a member of the College of Science and Technology, Nottingham Trent University, Nottingham, U.K. and Intelligent Systems Research Centre, Ulster University, Londonderry, U.K.

S. Coleman is a member of the Intelligent Systems Research Centre, Ulster University, Londonderry, U.K.

Manuscript received Month Day, Year; revised Month Day, Year. auditory pathway. This adaptation has been observed experimentally in animal studies with the site of the hyperactivity localized to the dorsal cochlear nucleus (DCN) [12], [13], [14], [15], [16], [17]. The biological literature [18], [19], [20], [15] has shown that the location of neuronal hyperactivity in the tonotopically organized DCN corresponds to the tinnitus pitch, furthermore the intensity of this hyperactivity correlates with the subjective tinnitus loudness. Therefore it should be possible to infer the perceived tinnitus pitch and loudness from the hyperactivity as the neuronal activity within the DCN during tinnitus perception, resembles sound evoked activity. Human studies have observed changes in the cochlear nucleus activity through auditory brainstem response (ABR) recordings [9], [21]. In [9] a hyperactivity model is used to try to explain the recorded ABR data. The increased spontaneous neuronal activity is frequency specific and localised to frequencies in and around regions of hearing loss [17]. The hyperactivity is maintained along the ascending auditory path with evidence of hyperactivity within the inferior colliculus [22], [23]. Moreover, this activity is proportional to the hyperactivity observed at the DCN [23]. At a cortical level this hyperactivity is observed in the auditory cortex [24], [25]. It should be noted that the amount of cortical hyperactivity is not necessarily proportional to tinnitus distress or perception [25]. It is at the cortical level where the activity in the auditory system is perceived as a phantom sound (so called 'phantom' as the activity does not arise from external auditory stimulation). Increased neural synchrony in the auditory cortex facilitates perceptual binding which manifests itself as sound despite the absence of auditory stimulus [26]. This phenomena is also apparent in the auditory brainstem. Therefore to model tinnitus perception it is important to model the activity within the DCN [5], [3]. Tinnitus-related activity has been observed in nonauditory areas associated with memory, attention and emotion (see [27], [28] for thorough reviews of the findings). Moreover, the neural substrate, and the functional connectivity between cortical regions is similar to that observed in people with neuropathic pain [29]. A review of the cortical and subcortical physiological changes associated tinnitus can be found [31]. Understanding the mechanisms of tinnitus is challenging due to the heterogeneity of the people who experience tinnitus as outlined in a recent review [30].

Computational models have been used to investigate the mechanisms responsible for tinnitus-related hyperactivity [32], [33], [34], [37], [38] with varying methodologies; namely lateral inhibition, gain adaptation and stochastic resonance being proposed. Although these studies model experimentally observable hyperactivity as a characteristic related to tinnitus, few models have benchmarked the modelled perception against measured data relating to the perceptual characteristics of tinnitus. Perceptual models of tinnitus have only focussed pri- 
marily on modelling tinnitus pitch and have not accounted for other perceptual aspects like loudness or other characteristics (for example pure-tone/ringing/hissing).

Computational models to date primarily focus on changes in the ascending, or bottom-up, auditory signals. The descending signals in the auditory pathway, or top-down signals, have also been shown to be crucial for the generation of tinnitusrelated activity [11], [39]. It is challenging however to directly measure the top-down signals in a non-invasive way. Few studies have been carried out to understand how tinnitusrelated activity leads to actual perception of a sound by an individual. A qualitative approach to tinnitus pitch prediction was carried out in [35], [32] while a quantitative approach was taken in [36]. These approaches showed that the bottomup activity provides sufficient information to enable a good approximation of the frequency of the sound perceived by the person with tinnitus, however the model prediction is not perfect.

Modelling of bottom-up adaptation in people with tinnitus has been deterministic [36], [32], [11], [53]. In other words the amount of adaptation has been proportional to the relative degree of hearing impairment with no stochastic element included in the models. This can be mathematically described by

$$
\text { perception } \sim \text { ascendingsignals }+\epsilon
$$

where only the ascending signals are used to describe the tinnitus perception plus some error, denoted $\epsilon$. Incorporating top-down signals in a model is challenging without a detailed knowledge of the biological mechanisms generating these signals the process is stochastic. More recent modelling approaches, such as stochastic resonance models [37] or perceptual updating models [38], incorporate feedback loops in to the modelled auditory system to dynamically respond to hearing impairment. It is known that the feedback signals impact on how people perceive sound. For instance, one person may perceive a sound to be louder than another person's perception of the same stimulus. The individual variability can be accounted for through 'random effects'. In this way it is possible to account for the effect top-down signals have on dependent samples and create a unique baseline for each subject. Although individuals may perceive ascending auditory signals differently there is at least consistency within each individual. The framework of a linear mixed effect (LME) model facilitates the modelling of fixed and random effects. Using such a model, tinnitus perception would be described in terms of the fixed/deterministic data, the ascending auditory signals, and the random effects, the idiosyncratic variation in perception that is due to individual differences.

This work aims to extend existing tinnitus models of hyperactivity to produce a perceptual model of tinnitus that accounts for pitch and loudness. The hypothesis is that perceptual modelling of tinnitus can be improved by accounting for the influence of top-down signals in the perception of a phantom sound. To do this, a previous tinnitus model [36] is extended and enhanced by biologically faithful modelling of the auditory periphery; named the modified periphery model. The perceptual modelling in [36] is used as a benchmark to compare the current state-of-the-art approach with the novel approach presented. A Linear Mixed Effects (LME) model is used to incorporate the influence of top-down signals on the perception of ascending auditory signals, while the performance of the modified periphery model and the LME model is compared with existing perceptual models of tinnitus.

Section 2 provides an overview of the data which are to be modelled in this work. Section 3 details the development of the modified peripheral model (MPM) and the LME model for tinnitus. Section 4 outlines the results of each part of the study before the results are discussed in Section 5. Finally, Section 6 concludes the paper with an overview of the findings from this work.

\section{DATASETS}

Two datasets are used in this study. Dataset 1 was previously used in the study of pitch prediction of tinnitus [36]. Dataset 2 , outlined in [40], is a slightly larger dataset which provides additional information pertaining to the perception of loudness which is not available in Dataset 1. Both datasets contain audiometric data and a frequency which best corresponds to the pitch of a person's tinnitus. During the collection of both datasets the challenges to accurately match the stimulus frequency to the person's tinnitus pitch have been acknowledged and measures were taken to ensure reliable data were recorded. Details of these measures can be found in the methodology of [41], [40]. Although there are minor differences in the audiometric data recorded in each dataset, detailed in Sections 2.1 and 2.2 , there is a significant overlap between the frequencies used and the range of frequencies considered. Moreover, both sets of data are fair representations of the audiometric and perceptual characteristics of the tinnitus population [7]. Since there is no objective measure available for perceptual properties of tinnitus, for example tinnitus pitch or loudness, the originators of both datasets have made conscious efforts to ensure the quality and integrity of the data collected. Details of these procedures are given in the original papers for each dataset [41], [40] while a description of each dataset is provided below.

\section{A. Dataset 1}

The first dataset was originally presented in [41] where the full details of the procedures used in the collection of the data can be found. This dataset was chosen as a subset of the full dataset which has been used in previous perceptual modelling studies for tinnitus [36]. Similarly, this study uses the same data as in [36] consisting of 48 sets of pure-tone audiometry and corresponding tinnitus pitch match for each ear of the participating 24 male subjects. Each subject had noise-induced hearing loss and perceive tinnitus as a tone like sound. Puretone audiometry was conducted for 10 frequencies $(0.125$, $0.25,0.5,1,1.5,2,3,4,6$ and $8 \mathrm{kHz}$ ). Subjects were asked to compare the most prominent tinnitus pitch they perceived with each of the 10 pure-tones used in the audiometry recordings and select one of these pure-tones which best matched their tinnitus pitch. 
Figure 1a shows the distribution of tinnitus pitches within the dataset. It is notable that the frequency is given per ear as one subject had a different dominant frequency in each ear. Figure 1 shows the audiometric data contained with in Dataset 1.

\section{B. Dataset 2}

The second dataset was originally presented in [40] where full details of the procedures used in the data collection can be found. The dataset used in the present work consists of 74 subjects with chronic stable tinnitus and come from the 'Hamilton' group reported in [40]. Audiometric tests were conducted at 17 frequencies $(0.1,0.3,0.5,0.8,1,1.5,2,3,4$, $6,8,9,10,11,13,14$ and $16 \mathrm{kHz}$ ) and subjects were asked to compare the dominant tinnitus pitch with 11 different sounds. The similarity of each sound to a person's tinnitus was subjectively rated using a logarithmic Borg CR100 psychophysical scale ranging from 0 to 100 where 100 corresponds to an exact match and 0 corresponds to no resemblance. The 11 sounds were centred around $0.5,1,2,3,4,5,6,7,8,10$ and $12 \mathrm{kHz}$ and were then modulated to reflect a pure-tone, ringing sound or hissing sound in line with the person's tinnitus. The subjects were also asked to adjust the loudness of each of the 11 sounds until it matched the loudness of their tinnitus.

Figure $2 \mathrm{a}$ shows the distribution of tinnitus pitches within the second dataset. The pitches in this larger dataset are more evenly distributed than the pitches in dataset 1 and also cover higher frequency ranges. A single tinnitus pitch is captured for each subject corresponding to the centre frequency of the dominant sound heard by each subject unlike Dataset 1 which considered each ear separately.

\section{METHODS}

The first part of this section outlines the modified periphery model which extends a previous perceptual model [36] by including a detailed ear model [42] and a biologically faithful model of the auditory nerve (AN) fibres. The second part will outline the LME model which provides a novel approach to perceptual modelling of tinnitus. The new approach is proposed to account for variability across subjects brought about by the uniqueness of individual sound perception. This section concludes with the definition of the metrics which will be used to evaluate the performance of each model.

\section{A. Development of peripheral model}

Figure 3 provides an illustration of the structure of the proposed MPM comprising of a model of the auditory periphery (the peripheral model) and a layer of laterally inhibiting neurons at the output end of the model to enhance tinnitusrelated hyperactivity and assist with pitch prediction [36]. The peripheral model begins with a biologically inspired ear model whose input is an instantaneous pressure waveform and whose output is the mean firing rate over time of a single AN fibre with a given characteristic frequency [42]. The tonotopic map for Dataset 1 is modelled between $125 \mathrm{~Hz}$ to $8000 \mathrm{~Hz}$ using a 0.35 filter spacing on the Equivalent Rectangular
Bandwidth (ERB) scale to approximate the bandwidths of the filters in human hearing [43]. The extended audiogram readings in Dataset 2, up to $12 \mathrm{kHz}$, enable the tonotopic map for this dataset to be modelled over more frequencies whilst maintaining the same ERB spacing as Dataset 1. As the tonotopic mapping is known to exist from the cochlea through to the auditory cortex, there is a canonical one-to-one mapping of frequencies between each module of the peripheral model and through to the lateral inhibition layer (Figure 3 ). To model hearing damage, the inner and outer hair cell parameters of the ear model are adapted using an audiogram fitting function available with the open sourced ear model [44]. Full details of how hearing impairment adjusts the hair cell related parameters of the ear model can be found in [45]. The remainder of the ear model parameters are unchanged from [42].

The ear model depicts low, medium and high spontaneous AN fibres; that is, those AN fibres with low spontaneous firing rates $(\leq 10 \mathrm{~Hz})$, AN fibres with a medium spontaneous firing rates $(\leq 20 \mathrm{~Hz})$ and $\mathrm{AN}$ fibres with a high spontaneous firing rate $(\geq 20 \mathrm{~Hz})$. The number of AN fibres per characteristic frequency in the tonotopic map of each dataset is inferred using the work of Greenwood to determine the proportion of the 30000 AN fibres that would be found at each characteristic frequency in humans [46]. The proportion of low, medium and high spontaneous fibres is $15 \%, 25 \%$ and $60 \%$ respectively and it is assumed that this distribution is uniform across frequencies [47]. It is possible that the distribution is non-uniform in humans as found in other mammals [48], [49] however without the precise distribution of fibres across frequencies in humans the decision is made to keep it consistent rather than to speculate. This assumption of uniformity is discussed further in Section V. Weighted by the proportion of each fibre type and the number of AN fibres per frequency, the AN firing rates are summed into a single mean firing rate over time for each characteristic frequency. The overall net activity, $f_{c}$, of the AN fibres at a particular frequency, $c$, is modelled by

$$
f_{c}=\frac{p}{q}\left[\begin{array}{lll}
0.6 & 0.25 & 0.15
\end{array}\right]\left[\begin{array}{lll}
h & m & l
\end{array}\right]^{T}
$$

where $p$ is the number of $\mathrm{AN}$ fibres that contribute to the net activity of the $\mathrm{AN}$ at a given frequency and $q$ is the total number of fibres per characteristic frequency. The first vector in Equation 2 represents the distribution of the high, medium and low spontaneous fibres (high $=60 \%$, medium $=25 \%$ and low $=15 \%$ ). The firing rates of the high, medium and low spontaneous fibres are denoted as $l, m$ and $h$ respectively. For convenience, we use the notation $f_{c}$ rather than $f_{c}(t)$. The AN fibres provide input to the DCN module.

The architecture for the DCN model comes from [50] and is characterised by three populations of neurons; namely wideband inhibitor (WBI) neurons, narrowband inhibitor (NBI) neurons and projector neurons (PNs). The individual neurons are modelled as in the Schaette and Kempter tinnitus model [34] and the parameter values are consistent with this study. Although the AN model used here provides an analogous output to the AN model used in [34], the use of a dynamic AN model in this work (in contrast to the firing rate $\mathrm{AN}$ 


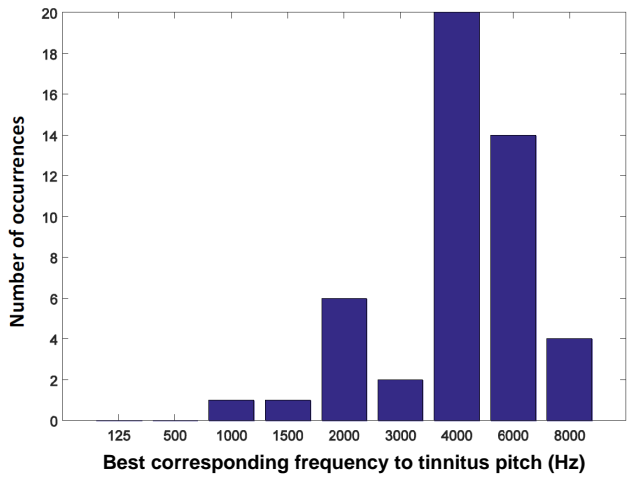

(a) The distribution of best corresponding frequency to tinnitus pitch across Dataset 1 (the $y$-axis is given in terms of ears)

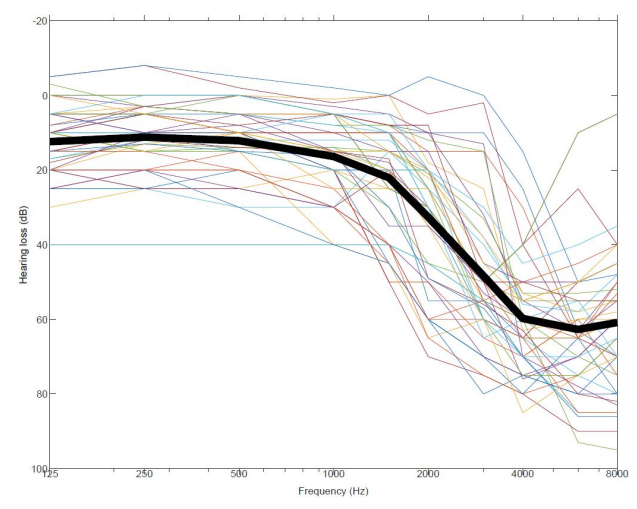

(b) Illustration of the audiograms contained in Dataset 1 with the mean hearing threshold shown in bold

Fig. 1. Illustration of Dataset 1 showing (a) the recorded tinnitus pitches and (b) the audiometric data.

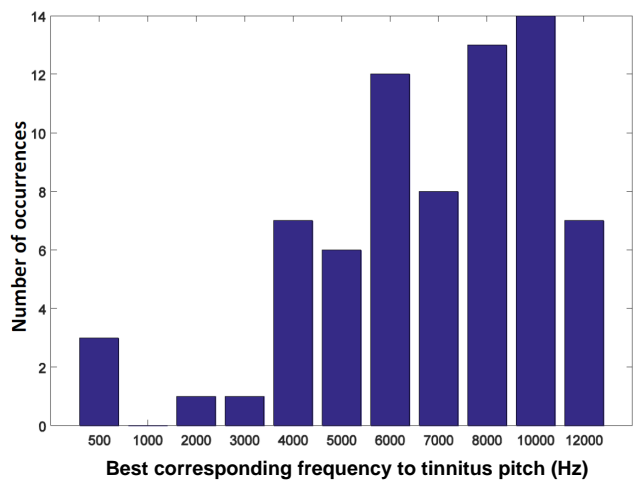

(a) The distribution of best corresponding frequency to tinnitus pitch across Dataset 2 (the $y$-axis is given terms of subjects)

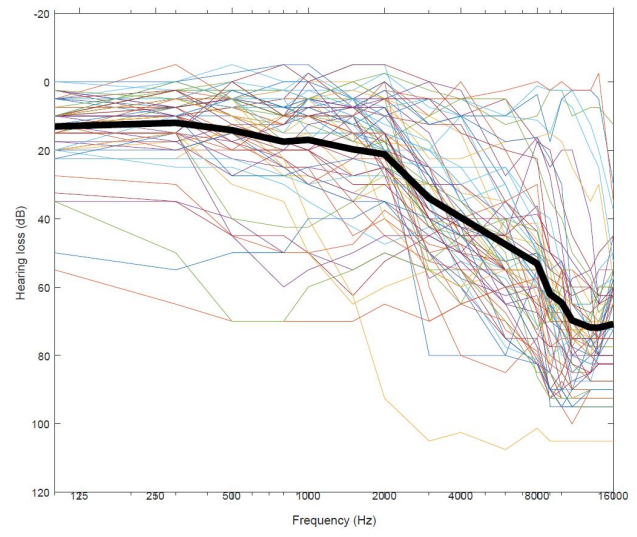

(b) Illustration of the audiograms contained in Dataset 2 with the mean hearing threshold shown in bold

Fig. 2. Illustration of Dataset 2 showing (a) the recorded tinnitus pitches and (b) the audiometric data.

model in [34]) means that some of the subsequent equations in the Schaette and Kempter tinnitus model [34] are altered. Consequently the dynamics of the WBI and NBI neurons and PNs are outlined below. The firing rate, $f_{c}$, of an AN fibre for a given characteristic frequency, $c$, provides excitatory input to the WBI and NBI neurons and PNs. The firing rate, $w_{c}$, of a WBI neuron of characteristic frequency $c$ is

$$
\begin{aligned}
w_{c} & =W\left(f_{A}, \ldots, f_{B}\right) \\
& =\left[\frac{1}{N} \sum_{\substack{i=A \\
i \neq c}}^{B} f_{i}-\theta_{i}^{w}\right]_{+}
\end{aligned}
$$

Here frequencies $A, B$ and $c$ satisfy that $c \in\{A, \ldots, B\}$ and $|\{A, \ldots, B\} \backslash\{c\}|=N=10 \%$ of the total size of the tonotopic map for each dataset. The positive rectifier is denoted by $[\ldots]_{+}$ and defined by $[x]_{+}=\max (0, x)$. The firing threshold of the WBI neurons, $\theta_{i}^{w}$, is chosen such that $f_{i}-\theta_{i}^{w}=0$ when frequency $i$ is stimulated by a sound less than or equal to $27 \mathrm{~dB}$ in line with previous models [34]. Therefore WBI neurons are inactive until there is broadband noise greater than $27 \mathrm{~dB}$. The choice in threshold means that WBI neurons will not be unnecessarily stimulated by spontaneous neuronal activity or quiet environments while the threshold remains low enough to allow for low level sounds, such as a whisper $(\approx 30 \mathrm{~dB})$, to be processed.

The NBI neurons are stimulated by pure tones and are inhibited when there is broadband noise. The firing rate, $n_{c}$, of the NBI neurons with characteristic frequency $c$ is given by

$$
n_{c}=N\left(f_{c}, w_{c}\right)=\left[g_{f} f_{c}-g_{n w} w_{c}-\theta_{c}^{n}\right]_{+}
$$

where the synaptic weight between the WBI neurons and NBI neurons, $g_{n w}=1.5$ (as in [34]), ensures that NBI neurons do not respond to broadband noise. The gain factor for the excitatory AN input is $g_{f}=1$. The firing threshold $\theta_{c}^{n}$ is equal to $\theta_{c}^{w}$ (Equation 3) so that the NBI neurons respond to pure tones in the same way as WBI neurons respond to broadband noise.

The PNs are the final layer in the DCN module within the peripheral model. They receive input from the AN fibres, WBI 


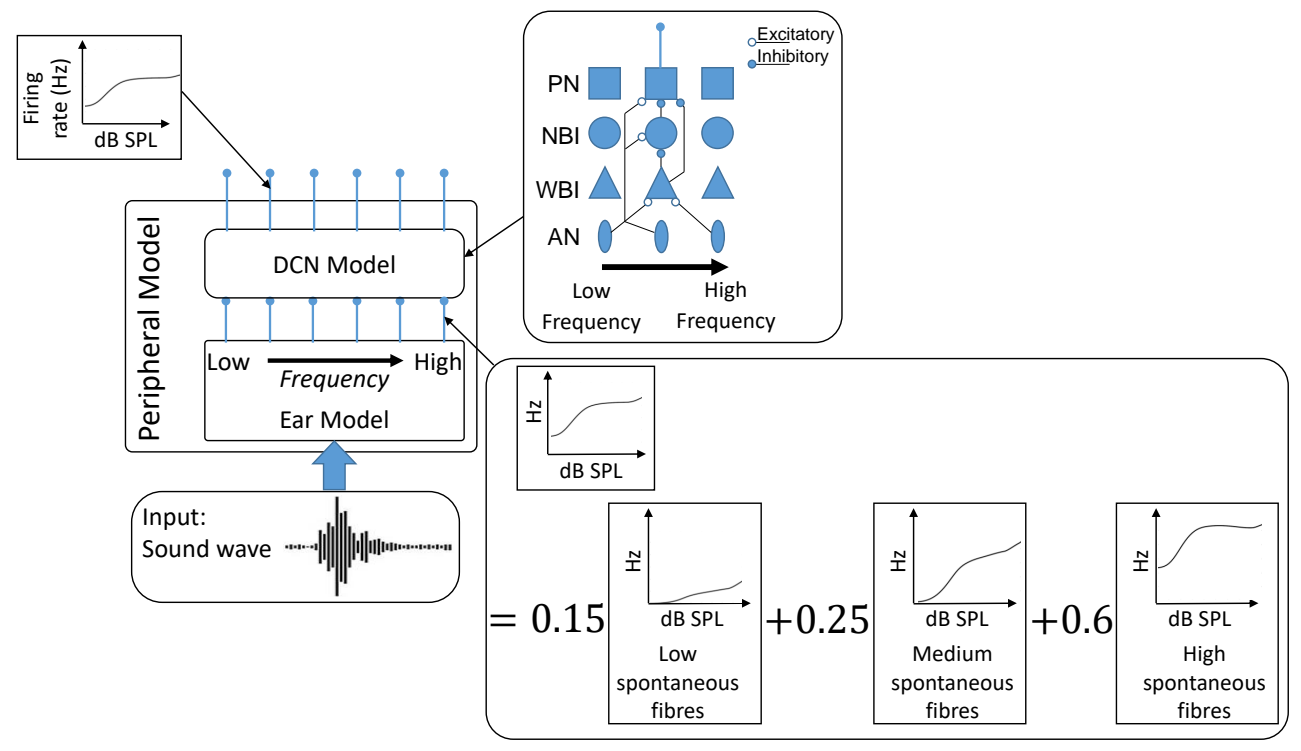

Fig. 3. Topology of the MPM [WBI $=$ wideband inhibitor, NBI $=$ Narrowband inhibitor and $\mathrm{PN}=$ projector neurons $]$. The rate intensity functions illustrated for the low, medium and high spontaneous fibres, auditory nerve fibre and PN output are taken from the model using a characteristic frequency of $1 \mathrm{kHz}$ and a intensity range of $[0,90] \mathrm{dB}$ sound pressure level (SPL).

and NBI neurons. The firing rate of a PN is calculated by

$$
\begin{aligned}
r_{c} & =R\left(f_{c}, w_{c}, n_{c}\right) \\
& =r_{h i g h} \tanh \left(\frac{\left[g_{f} f_{c}-g_{w} w_{c}-g_{n} n_{c}\right]_{+}}{r_{h i g h}}\right)
\end{aligned}
$$

where $r_{\text {high }}=300$ (consistent with [34]). The values of the gain factors $g_{w}$ and $g_{n}$ can vary the dynamics of the neuron model as explored in [34]. It is within the DCN model that homeostatic plasticity is modelled to adapt the gain factors in Equation 5.

After hearing damage, such as sensorineural hearing loss, the AN fibres will decrease in their spontaneous and maximum firing rates. Consequently, the input to the PNs is decreased lowering the mean firing rate of these neurons [9]. To define the mean firing rate of the PNs, in line with previous studies [34], [36], we must first define a number of components. The probability density function of the sound intensity $I$ (in $\mathrm{dB}$ ) given by

$$
p_{I}(I)=\frac{1}{\sqrt{2 \pi \sigma^{2}}} \exp \left(-\frac{(I-\mu)^{2}}{2 \sigma^{2}}\right)
$$

where the mean intensity is $\mu=40 \mathrm{~dB}$ with standard deviation $\sigma=25 \mathrm{~dB}$. Given a hearing threshold of $T \mathrm{~dB}$ for a particular frequency the probability of spontaneous activity, $P_{s p}$, occurring at the frequency is defined as

$$
P_{s p}=\int_{-\mathrm{inf}}^{T} p_{I}(I) \cdot d I
$$

The probability distribution $p_{f}$ of the AN firing rates is shown in Figure 4. The probability distribution estimate was obtained using the ksdensity function in MATLAB with a normal kernel smoother. The probability density function can be modelled as the sum of two Gaussian distributions with mean 78 and 202 respectively and standard deviation 32 and 120 respectively.
The values for the spontaneous and maximum firing rates are obtained by stimulating the AN fibre with a pure tone centred at the characteristic frequency of the AN fibre at $0 \mathrm{~dB}$ for spontaneous firing rate and $85 \mathrm{~dB}$ for maximum firing rate. The maximum sound level of $85 \mathrm{~dB}$ is chosen because it would not be practical or ethical to validate this model's behavior through electrophysiological studies which would stimulate the ear at very high sound intensity, for example, $120 \mathrm{~dB}$. The lower sound level could be used in future real-world validation of the model which would not be practical or ethical with a very high sound intensity. The mean firing rate of the PNs is defined by

$$
\bar{r}=\int_{f} p_{f}(f) R(f) \cdot d f
$$

In order to adapt to the reduced mean firing rate the gain within the PN model is adjusted. By altering Equation 5, the gain factor, $g$, stabilises the mean firing rate such that the firing rate of a PN can now be written as

$$
\begin{aligned}
r_{c} & =R\left(f_{c}, w_{c}, n_{c}\right) \\
& =r_{h i g h} \tanh \left(\frac{\left[g f_{c}-\frac{g_{w}}{g} w_{c}-\frac{g_{n}}{g} n_{c}\right]_{+}}{r_{h i g h}}\right) .
\end{aligned}
$$

The value of $g$ is calculated numerically using the Secant method and is bounded between 1 and 3 inclusive in keeping with previous models [34]. The firing rates from the PN layer provide the input to the lateral inhibition layer of neurons which will be used to extract the tinnitus pitch from the modelled auditory activity.

The output of the PNs provide the activity of each frequency modelled in the tonotopic map from which a prediction of the pitch of the tinnitus sound will be made. To enhance peaks along the tonotopic map and suppress noise, the last stage of the MPM is a layer of neurons that mutually inhibit 

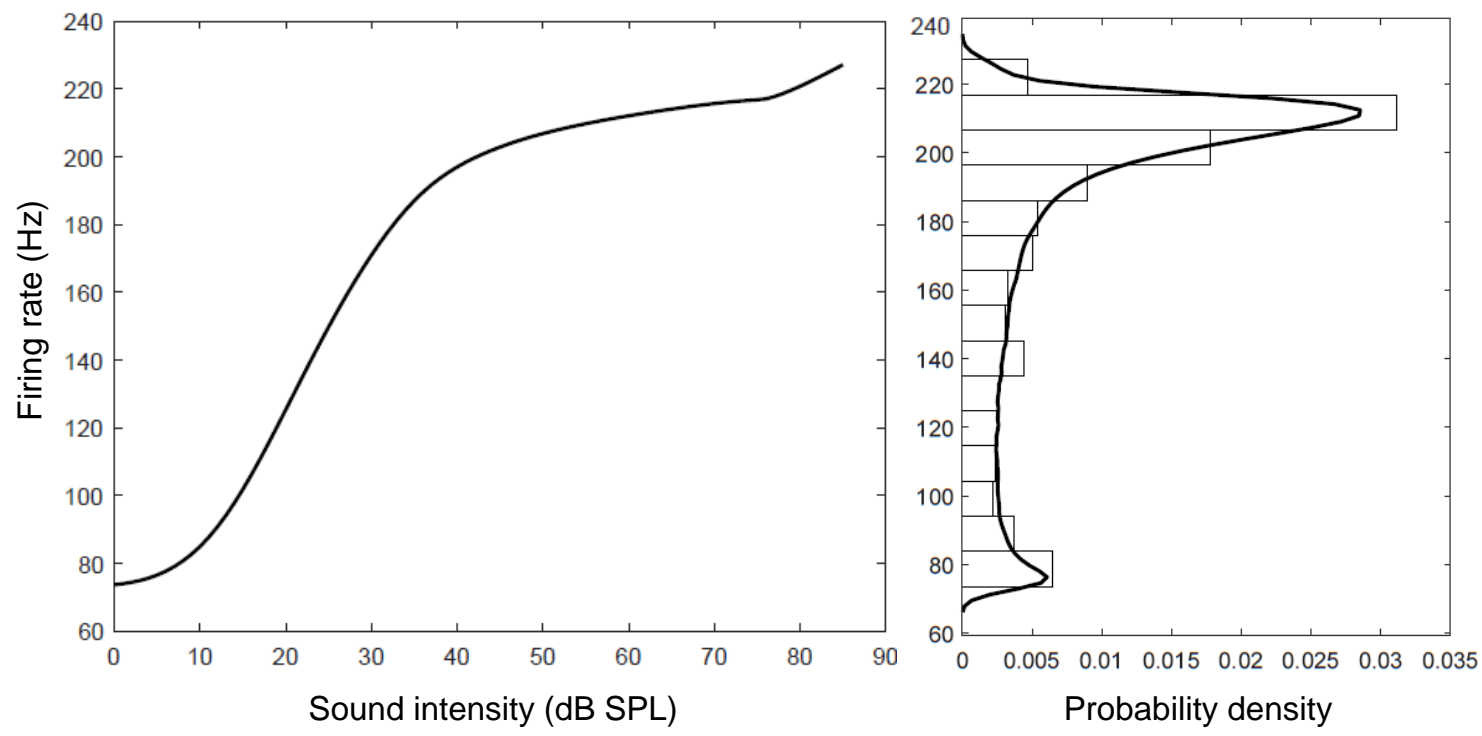

Fig. 4. The AN firing rate (left) over sound intensity of stimulus at the characteristic frequency of the AN (1kHz shown here). The probability density function for the AN firing rate (right) is characterised by a bimodal distribution.

neighbouring neurons. The output of the lateral inhibition layer of neurons is defined, as in [36], by

$$
a(t)=[r(t)+M \cdot a(t-1)]_{+}
$$

where $r(t)$ is the output of the projector neurons in the DCN model, $M$ is the matrix of lateral inhibitory weights and $a(0)=$ 0 . The inhibitory weight between the $i^{t h}$ and $j^{\text {th }}$ neurons is

$$
a= \begin{cases}\frac{-w_{\max }}{2} \cdot\left(1+\cos \left(\pi \frac{i-j}{\sigma}\right)\right) & \text { for }\|i-j\| \leq \sigma \\ 0 & \text { otherwise }\end{cases}
$$

where, in [36], $w_{\max }=0.8$ and $\sigma=5(\sigma=10$ for the Gerken model [51]) is the width of the inhibitory neighbourhood. Both of the previously stated values for $\sigma$ will be considered in turn. Although only a single lateral inhibition layer is illustrated in Figure 3, the recursive nature of Equation 10 means that modelling lateral inhibition over $T$ time steps is equivalent to a static lateral inhibition model with $T$ layers comparable to previous lateral inhibition models used in tinnitus modelling [51], [52], [53]. The predicted pitch is taken to be the frequency of the neuron in the lateral inhibition layer with the greatest activity at the end of the simulation. An analogous approach was used in [36] however the number of time steps used in the simulations has not been given in [36]. An arbitrary value of $T=10$ seconds is chosen in the first instance. In Section 4 it will become apparent that the values of $w_{\max }, \sigma$, and $T$ will require refinement.

\section{B. Capturing heterogeneity in the tinnitus population}

The approaches of qualitative perceptual models of tinnitus [32], [35] and the quantitative approach in [36] (and analogously the methodology above) assume that the perceived tinnitus pitch could be determined from the bottom-up signals in the auditory brainstem (weighted to optimise the results) plus some error. This methodology is similar to the approach of linear models which take the form

$$
y=X \beta+\epsilon
$$

where the observed data, $y$, are calculated using a matrix of fixed effects, $X$, weighted by scalars, $\beta$, with a generic error, $\epsilon$. In the context of tinnitus pitch prediction, the observed data $y$ correspond to the perceived tinnitus pitches and $X$ contains quantitative variables for each subject that represent the fixed activity of the auditory brainstem. This model essentially separates the problem of pitch prediction into two parts. Firstly the known components, $X$, and secondly the error term for the components that cannot be captured, $\epsilon$. In this structure the error term is assumed to be general and be the same across all subjects. However, perception of bottom-up signals in the auditory system is unique across different people and is moderated by top-down signals in the descending auditory pathway. Therefore the addition of a random effect for each person is needed to capture the subtle variations across the population. One novel approach to do this is to extend the linear model in Equation 12 to a LME model.

The LME model is expressed as a mixture of fixed and random effects such that

$$
y=X \beta+Z b+\epsilon
$$

where $Z$ is the random effects matrix containing known quantitative variables weighted by scalars, $b$. This model requires some known information pertaining to the random element of the model, in this case the subject's individual perception of their tinnitus sound.

The subjective likeness ratings of known sounds relative to the perceived phantom sound in Dataset 2 will be used as the known random effects in the LME model denoted by $Z$ in Equation 13. The rows of $Z$ correspond to different subjects while the columns correspond to different known sounds used 
for the subjective likeness ratings. As each individual will have a different way of subjectively rating the likeness of the sound stimuli (i.e. one person's rating of 50/100 may be another person's rating of 60/100 for the same stimuli), these data are specific to each individual. Therefore, the random effect component of the model provides a unique baseline for each individual subject. As it is not possible to passively measure the exact auditory nerve activity at each frequency of the tonotopic map, the ascending auditory signals will be depicted using the modelled activity of the peripheral model. The output of the PNs in the DCN will provide the data for the fixed effects matrix $X$ in Equation 13.

The LME model will be used to model both the pitch and loudness of a person's tinnitus. The fixed effects matrix of the LME model will contain the activity of the PNs in the peripheral model for both the pitch and loudness modelling cases. This activity contains information regarding the pitch and loudness of a sound. The pitch is relevant to the tonotopic map and the location of hyperactivity within the tonotopic map. Loudness is the perception of the volume of sound stimulus. The firing rate of neurons along the auditory brainstem is directly proportional to the loudness of the sound [54].

The scaling parameters $\beta$ and $b$ of the LME model are fitted across the entire dataset using the fitlmematrix function in MATLAB with the maximum likelihood approach. The optimisation of the model fit is carried out using a quasi-Newtonian approach (using the optimisation function quasinewton). The predicted values are obtained by applying the MATLAB function fitted to the LME model so that quantitative analysis could be performed on the model's performance.

A stepwise forward selection approach is used to establish which random effects and, indeed, how many random effects should be included in the model. The quality of each model is determined by the Akaike information criterion (AIC) which takes in to account the trade-off between the simplicity of the model and the goodness of fit. In the first instance the LME model is fitted with no random effects. In the next step, all permutations of a single random effect parameter are considered. If the minimum AIC for models containing one extra parameter is less that the AIC for no random effects parameters then the addition of another parameter is considered. The next step considers all permutations of the remaining random effects alongside the random effect parameter from the previous step. While the minimum AIC for all models with the given number parameters is less than the previous step the process of adding an extra parameter is considered. The final LME model is the model with the lowest AIC and therefore representing the best compromise between model simplicity and goodness of fit. This approach is used to ensure the LME model is not become overfit to the dataset.

\section{Quantitative measures of perception prediction}

In line with previous perceptual modelling of tinnitus [36] the performance of a model for predicting tinnitus pitch will be evaluated in terms of the error, the model bias and the correlation between the model's predicted pitches and the perceived pitches of people's tinnitus from Dataset 1 and Dataset 2.

The error in the model's performance is defined in terms of the root-mean-square error (in octaves) of the predicted tinnitus pitch $p_{i}$ (for each sample $i$ of each dataset) and the perceived tinnitus pitch $t_{i}$ as determined experimentally. That is to say, the error $E$ is given by

$$
E=\sqrt{\frac{1}{n} \sum_{i=1}^{n} \log _{2}\left(\frac{p_{i}}{t_{i}}\right)^{2}}
$$

where $n$ is the size of the dataset. The model bias (in octaves), $B$, is defined in terms of the difference between the mean of $p, \bar{p}$, and the mean of $t, \bar{t}$. That is

$$
\begin{aligned}
B & =\frac{1}{n} \sum_{i=1}^{n} \log _{2} p_{i}-\frac{1}{n} \sum_{i=1}^{n} \log _{2} t_{i} \\
& =\bar{p}-\bar{t} .
\end{aligned}
$$

The correlation, $C$, between the perceived pitches and the predicted pitches is determined by

$$
C=\frac{\operatorname{Cov}(p, t)}{\sigma_{p} \sigma_{t}}
$$

where the covariance is defined as

$$
\operatorname{Cov}(p, t)=\frac{1}{n-1} \sum_{i} \log _{2} p_{i} \cdot \log _{2} t_{i}-\bar{p} \cdot \bar{t}
$$

and $\sigma_{p}$ and $\sigma_{t}$ are respectively defined as

$$
\sigma_{p}=\sqrt{\frac{1}{n-1} \sum_{i}\left(\log _{2} p_{i}-\bar{p}\right)^{2}}
$$

and

$$
\sigma_{t}=\sqrt{\frac{1}{n-1} \sum_{i}\left(\log _{2} t_{i}-\bar{t}\right)^{2}} .
$$

In the case of modelling tinnitus loudness, only the correlation between the predicted loudness values and the perceived loudness values is used to evaluate the performance of the LME model. The MPM and LME model were implemented and the subsequent analyses of each model were implemented in MATLAB R2018a (The MathWorks, Natick, MA, USA).

\section{RESULTS}

The first investigation evaluates the performance of the MPM with Dataset 1 . Using the parameters of $w_{\max }=0.8$, $\sigma=5$ and $T=10$, as shown in [36], the error, bias and correlation when modelling Dataset 1 were 1.0422 , -0.7887 and 0.3702 respectively. When adjusting $\sigma=10$ as outlined in [36] to be the parameter settings for the Gerken model there is no significant change in any of the evaluation metrics. As this modelling attempt is substantially poorer than the original Schaette and Kempter perceptual (SKP) model [36] and but slightly better than the Gerken model [51] (evaluated in [36]) model, efforts were made to improve the parameters of the lateral inhibition layer. The values for $w_{\max }$, and $\sigma$ from Equation 11, and the number of time steps, $T$, were optimised using a Genetic Algorithm (GA) such that the error between the predicted pitch and the perceived pitch is minimised. The 
fitness function for the GA was constructed to calculate the error between the predicted pitch and the perceived pitch. The values of $w_{\max }, \sigma$ and $T$ are bounded by $(0,10),[2,64]$ and $[1,100]$ respectively with $\sigma$ and $T$ further constrained to be integers. The GA was implemented using the default parameters, i.e. a scattered crossover function and a Gaussian mutation function, of the built in ga function in Matlab R2018a (The MathWorks, Natick, MA, USA). The optimum parameter values of the lateral inhibition layer were calculated by the GA to be $w_{\max }=0.09, \sigma=12$ and $T=42$. The optimisation of the parameters of the MPM resulted in an improvement to the error, bias and correlation as documented in Table I.

Table I shows the performance of pitch prediction of each model for the given dataset alongside the results of existing perceptual models of tinnitus as reported in [36]. The error, bias and correlation of the MPM lies between the previous SKP and Gerken models as analysed in [36]. The findings are also in keeping with [36] which found the combination of gain adaptation and lateral inhibition, used in the SKP and MPM models, provides a more accurate model of tinnitus perception than lateral inhibition alone, as used in the Gerken model.

TABLE I

RESULTS OF PITCH PREDICTION FOR ALL MODELS

\begin{tabular}{l|l|l|l|l}
\hline Model & Dataset & Error & Bias & Correlation \\
\hline SKP & 1 & 0.59 & -0.05 & 0.52 \\
Gerken & 1 & 1.62 & -1.48 & 0.37 \\
MPM & 1 & 0.99 & 0.75 & 0.26 \\
& 2 & 1.24 & -1.60 & 0.39 \\
LME model & 2 & 0.22 & 0.02 & 0.97 \\
\hline
\end{tabular}

Figure 5 shows the model's predictions against the perceived pitch values. Note that any points lying on the identity line (i.e recorded pitch $=$ modelled pitch) were predicted to be the recorded perceived pitch of the person's tinnitus. The error in this model is significantly better than the Gerken tinnitus model [51] which was evaluated in [36] but performs marginally worse than the SKP model.

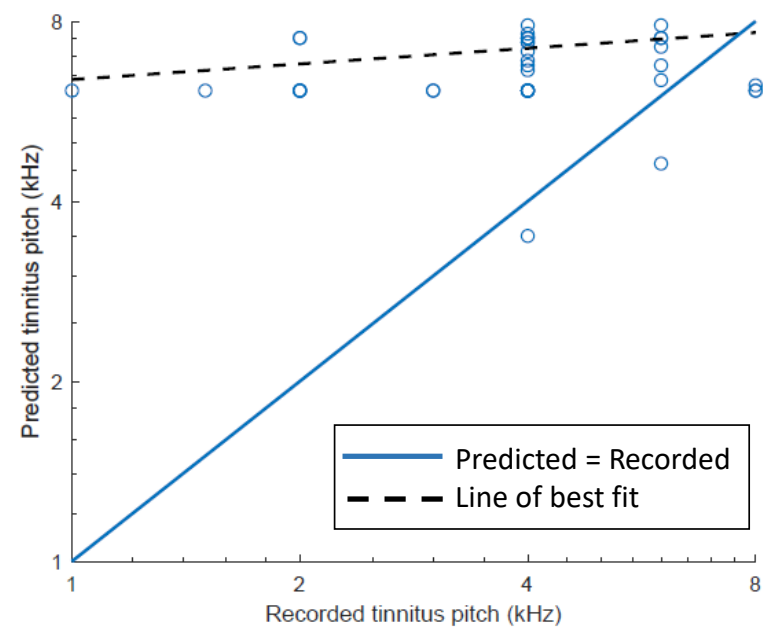

Fig. 5. Results of pitch prediction using the MPM and Dataset 1

The MPM was then applied to Dataset 2 (Figure 6). Using the SKP lateral inhibition parameters of $\sigma=5, w_{\max }=0.8$ and
$T=10$ the model performance was poor with $E=1.4237, B=$ -0.8340 and $C=0.3806$. Similarly, assigning $\sigma=10$, in line with [51], gave no improvement in any metric. As with Dataset 1 , the decision was made to optimise these three parameters to improve model performance. Following optimisation using a GA, the parameters of the lateral inhibition layer of the MPM using a GA were $\sigma=6, w_{\max }=0.17$ and $T=56$; the results are given in Table I. The MPM model performs better than the Gerken model according to all three metrics. However it performs worse with Dataset 2 than with Dataset 1. This can be explained by the distribution of pitches in Dataset 1 where the majority of samples had a pitch between $4000 \mathrm{~Hz}$ and $6000 \mathrm{~Hz}$. Therefore the model was still able to perform well as on average a prediction of $4000 \mathrm{~Hz}$ or $6000 \mathrm{~Hz}$ would result in a good prediction. This highlights that the error and bias of the model may not be strong indicators of the model's overall performance.

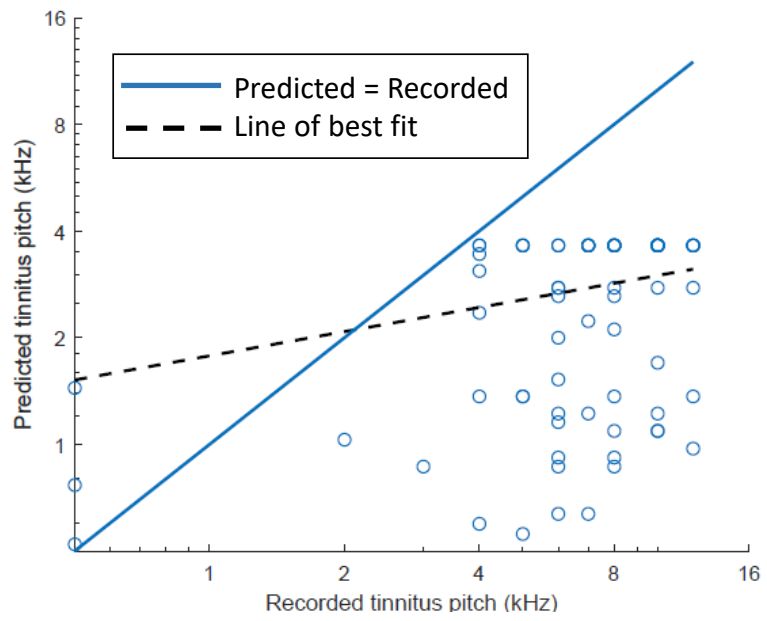

Fig. 6. Results of pitch prediction using the MPM with Dataset 2

The error and bias metrics are heavily affected by the values of the empirical data compared with the modelled data. In other words, these metrics reward very close model predictions and are very punishing on weaker model predictions. In contrast, the correlation reflects the model's performance as a whole over the entire dataset thus providing a better evaluation of how the model performs. The correlations between the modelled data by the MPM and Dataset 1 and 2 were 0.26 and 0.39 respectively indicating moderate correlation. The consistency of the correlation metric and lack of consistency in the error, and to a lesser extent the bias, across datasets highlights the appropriateness of the correlation metric to evaluate model performance. The results indicate that the MPM is not a reliable model of tinnitus perception as validated by the two independent datasets. This also highlights that tinnitus percept is not solely encoded by ascending auditory signals which the MPM represents.

The LME model can only be applied to Dataset 2 as it requires both fixed and random effects. The LME model was fitted using the built in MATLAB function fitlmematrix with default parameters. Figure 7 shows the LME model's predictions against the perceived values of the tinnitus pitch. As 
outlined in Table I, the error in the LME model's performance is $E=0.22$, the bias is $B=0.02$. The correlation between the LME model's predictions and the perceived pitch values is $C=0.97$ indicating a very strong correlation. This is clearly evident in Figure 7 which is greatly improved compared with all other models of tinnitus pitch in terms of the model's correlation with reality.

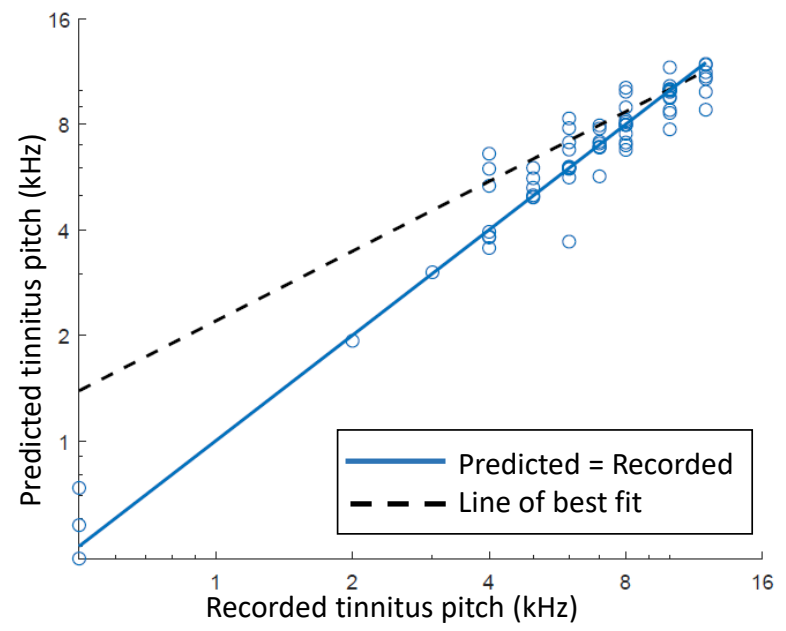

Fig. 7. Modelled tinnitus pitch against the recorded tinnitus pitch in Dataset 2 using the LME model

The optimum model from the forward selection approach includes four random effects. The random effects correspond to the subjective likeness rating of $500 \mathrm{~Hz}, 6 \mathrm{kHz}, 10 \mathrm{kHz}$ and $12 \mathrm{kHz}$ compared with the person's perceived tinnitus pitch. This model had the lowest AIC measure and the addition of more random effects had a negative impact on the quality of the model. Further analysis was conducted to identify any frequency specific patterns that may reflect potential underlying biological processing. The normalised fitted weights are shown in Figure 8. The recorded tinnitus pitches from the dataset are also marked along this function to highlight the frequency markers that are relevant to Dataset 2. It is apparent at $2 \mathrm{kHz}$,

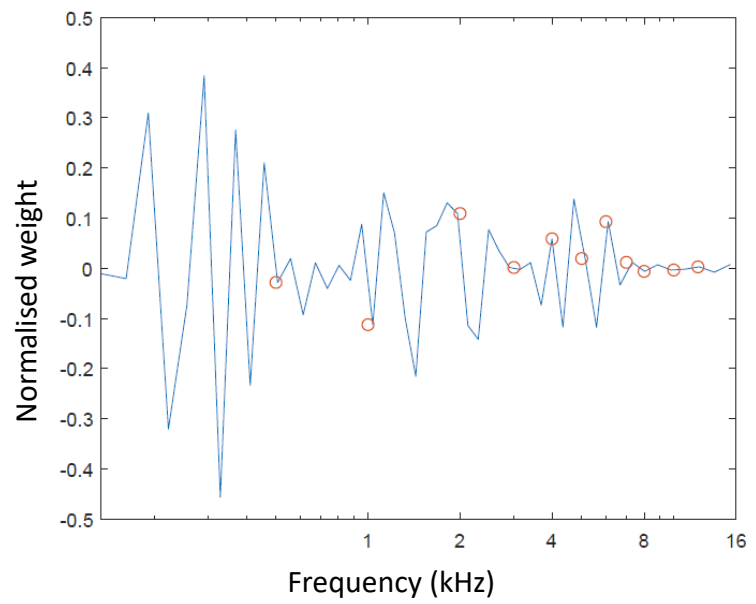

Fig. 8. The normalised weights of the fixed effects matrix of the LME model. The recorded tinnitus pitches from Dataset 2 are indicated by the red circles.
$4 \mathrm{kH}, 6 \mathrm{kHz}, 7 \mathrm{kHz}$ and to a lesser degree at $12 \mathrm{kHz}$ that the LME weights are near to a local maxima while the neighbouring frequencies receive a negative weighting. This behaviour is similar to localised lateral inhibition where some frequencies are amplified whilst neighbouring frequencies are suppressed. However, this behaviour is not apparent for every recorded tinnitus pitch; for example at $1 \mathrm{kHz}, 8 \mathrm{kHz}$ and $10 \mathrm{kHz}$. An independent evaluation would be needed using a secondary, and ideally, larger dataset to eliminate any potential dataset specific bias. It is also notable that lower frequencies receive a stronger weight, in general, than frequencies beyond $8 \mathrm{kHz}$. This may be explained by the fact that the activity of higher frequencies has previously been amplified in the DCN model in response to high frequency hearing loss.

The last stage of the investigation considers the novel modelling of tinnitus loudness. Figure 9 illustrates the LME model's predictions of tinnitus loudness against the perceived tinnitus loudness. The correlation between the LME model's predictions and the perceived loudness is $C=0.90$. This again indicates a strong correlation between the LME model's predictions and reality which is clearly evident in Figure 9. It is not appropriate to use the error and bias metrics in this case as their formulation is specifically related to pitch. The optimum LME model for loudness contains only two random effects. These parameters relate to the subjective rating of loudness of $3 \mathrm{kHz}$ and $7 \mathrm{kHz}$ compared with the perceived loudness of the person's tinnitus. This model was also developed incrementally using the forward selection approach, as in the pitch model, and the AIC is used to evaluate the quality of the model.

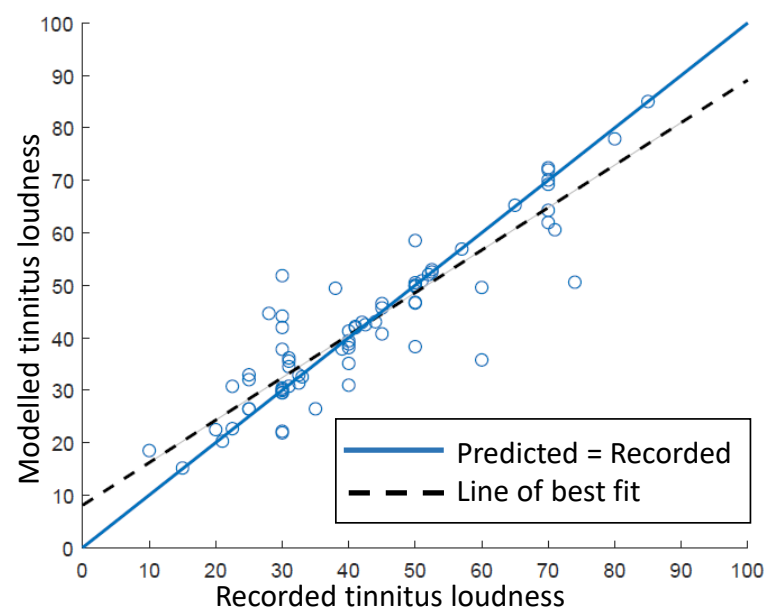

Fig. 9. Modelled tinnitus loudness against the recorded loudness of the tinnitus in Dataset 2 using the LME model

\section{Discussion}

To date tinnitus models have mainly focussed on replicating tinnitus-related activity in the auditory system. However it is important to go further to understand how a phantom sound is perceived following the development of hyperactivity in the auditory system. One challenge of tinnitus research is dealing with the uniqueness of the problem. This motivated the search for alternative methods which accounted for the heterogeneity 
of tinnitus perception by accounting for the influence of topdown signals which have been shown to be essential in the generation of tinnitus-related activity [11]. The LME model provides a structure to facilitate both fixed and random effects which contribute to the perception of pitch and loudness. The LME model accurately modelled the pitch and loudness of an individual's tinnitus and in doing so outperformed existing models of tinnitus perception with respect to correlation. This is the first perceptual model of tinnitus to consider the loudness of a person's tinnitus.

The LME had a higher accuracy for pitch perception than the MPM model. As discussed previously (in Section 2: Datasets), both datasets are fair representations of tinnitus perception. Therefore our postulate follows that the LME model provides the most accurate model of tinnitus pitch perception to date. Unfortunately this cannot be categorically proven in this work as it is not possible to apply the LME model to Dataset 1 nor is it feasible to apply existing perceptual models to Dataset 2 which would allow for a likefor-like comparison of tinnitus perceptrons. An explanation for the LME outperforming the MPM model, and possibly existing models, is that the MPM (and similarly the models outlined in [36] and [51]) can only account for ascending auditory information. In [11], [39] it has been shown that key neural correlates of tinnitus arise from top-down auditory modulation at the thalamocortical level. It is not feasible to directly observe these additional auditory signals in humans. However, the structure of the LME, in particular the random effects component, allows for unknown and person-specific bias of tinnitus perception to be accounted for by the inclusion of information pertaining to an individual's perception of known frequencies. The additional model complexity allows for increased model accuracy compared with existing models and the MPM model while it is not possible to directly account for all factors impacting on the tinnitus perception. The results of the LME model show that it is possible to accurately model an approximation of a person's perception of tinnitus using subjective likeness measures. The significant improvement in model performance (according to all three metrics) of the LME model from the MPM may show that the bottom-up activity may only have a moderate relation to the perceptual characteristics of tinnitus.

The fitted weights of the LME model, shown in Figure 8, may highlight the over simplicity of the MPM model whose behaviour is primarily adapted in response to hearing loss alone. It is therefore possible that the weights of the LME model reflect an aspect of the structural and/or functional aspects of auditory processing that is not captured by the simplistic MPM model used in this work. It is not possible to identify the origin(s) where such auditory processing may occur from the limited detail of the data available in this study. Moreover, the distribution of AN fibres is taken to be uniform across all frequencies where in reality it is highly possible that the distribution is non-uniform [48], [49]. Future work is required to capture more sophisticated data are needed to support the development of a more comprehensive model of the auditory system. This could include a more detailed modelling of hearing loss associated with hidden hearing loss. It may be possible to capture such data using electrophysiological approaches. Investigating treatment methods for tinnitus would be aided by the clarification of behaviour modelled by the fitted weights of the LME model.

The two LME models presented here, for pitch and loudness, only require a small number of random effects. In the case of pitch modelling, the random effects for $500 \mathrm{~Hz}, 6 \mathrm{kHz}$, $10 \mathrm{kHz}$ and $12 \mathrm{kHz}$ are selected through the forward selection approach to give the optimum model in terms of AIC. It is notable that these pitches do not correspond with the four most common pitches recorded in Dataset 2 (shown in Figure 2a) which are $10 \mathrm{kHz}, 8 \mathrm{kHz}, 6 \mathrm{KHz}$ and $7 \mathrm{KHz}$. Similarly for the LME loudness model which required random effects for information relating to the loudness of $3 \mathrm{kHz}$ and $7 \mathrm{kHz}$ stimulus. It is not immediately clear the significance of these random effects in terms of the underlying biological mechanisms. Future work to replicate our approach on a new, similar dataset would be beneficial in order to compare the findings with our current work. Unfortunately, such a dataset is not available at this time. Future experimental work, potentially through invasive electrophysiological recordings around the auditory brainstem, is needed to relate the subjective likeness ratings and the LME model parameters back to biological mechanisms. This may help explain the significance, if any, of the random effects included in the optimum LME models and the fitted weights discussed above.

This work investigated the spontaneous neuronal activity of people with tinnitus. In doing so this work, and previous investigations ([11], [36]), have under utilised the behaviour of the WBI and NBI neurons. The Carney ear model enables much richer stimuli to be investigated compared with previous AN models used to investigate tinnitus perception [36]. In future work, the MPM could be used to explore the role of the DCN model in more detail. Stimuli may include sounds commonly used in sound therapy approaches that aim to alleviate tinnitus therefore exploring the impact they may have on the perception of tinnitus pitch and loudness. Other realistic stimuli could be used to investigate the perception of tinnitus pitch and loudness in different acoustic environments. Future work would be needed to collect new data that could provide the ground truth to such an investigation.

The accuracy of the LME model means that the LME model can be used to objectively evaluate a person's subjective characterisation of their tinnitus sound. Until now tinnitus has been the individual perception of a sound which could not be accessed. This work unlocks the previously unobservable bottom-up signals and perception of a phantom sound. Moreover, the model accurately links the underlying mechanisms of tinnitus generation to the resulting perceived tinnitus sound. It is beneficial for clinicians to have a model of a person's tinnitus to not only help a person with tinnitus understand how the phantom sound originates but also to provide an accurate estimate of the phantom sound. This estimate of the phantom sound provides the clinician with a sound that they can hear allowing them to relate to the patient's problem and also reassures the patient that the clinicians do understand what the person is experiencing. This in turn provides confidence and encouragement to both the clinician and the patient. 
The results of the LME model, according to all three metrics used in the analysis, provides the most accurate perceptual model of tinnitus to date in terms of corresponding frequency and loudness. The MPM model performed mariginally worse than previous model work in terms of the correlation between the empirical data and the model's predictions. In a comparison to previous perceptual models of tinnitus outlined in [36], the choice of the model parameter $\sigma$, reflecting the span of the lateral inhibition of neighbouring frequencies, was similar to the optimised parameters of the MPM given in this study. The SKP model used $\sigma=5$ and the Gerken model used $\sigma=10$. In this study the MPM model used a value of $\sigma=12$ for Dataset 1 and $\sigma=6$ for Dataset 2. As noted in Section 3.1 these parameters were optimised with respect to the error of the modelled data from the empirical data. The inhibitory strength used in [36] was $w_{\max }=0.8$ whilst in this study the weaker strengths of $w_{\max }=0.09$ and $w_{\text {max }}=0.17$ were used for Dataset 1 and 2 respectively. Again, these parameters were determined by minimising the model error. These parameters are loosely coupled to the biological reality as it is not practical for any of the models discussed in this work to model the complex mechanisms involved at a cortical level for sound perception. Instead the aim is to extract relevant information, i.e. tinnitus pitch, in a practical way from a simplified model inspired by the known adaptation that exists in the auditory system of people with tinnitus. Hence, in this work the parameter values of the MPM are optimised to achieve maximal performance was in keeping with the previous models [36].

Three metrics were used to assess the performance of the MPM and LME models in line with previous investigations [36] of tinnitus pitch; namely the root-mean-square error (Equation 14), model bias (Equation 15) and the correlation between the perceived pitches and modelled pitches (Equation 16). The results outlined in Table I show that the MPM outperformed the Gerken model in terms of model error and model bias. However, it is evident by the correlation measure, and illustrated by the line of best fit in Figure 5, that the MPM is not a strong model of tinnitus pitch. As shown in Figures $1 \mathrm{a}$ and $2 \mathrm{a}$, the uneven distribution of tinnitus pitches in the datasets had a significant impact on the model's fit. The correlation metric accounts for the standard deviation in the empirical data and model's estimates as well as the covariance between the empirical data and model estimates. Thus this metric is less impacted by the skewed empirical data compared with the other two metrics. For this reason, we argue that correlation is the most appropriate metric of the three considered to evaluate model performance of tinnitus pitch prediction. Moreover it is also possible, for the same reason, to compare model performance across datasets using the correlation metric.

The methodology of the MPM and LME models presented in this work overlap between the different classes of existing tinnitus models. Fundamentally the MPM and LME are built on gain adaption models (i.e. the ear model and DCN model illustrated in Figure 3) analogous to those outlined in [11], [34], [35]. The lateral inhibition layer extends the gain adaption model within the MPM thus utilising the theory from previous lateral inhibition models of tinnitus [51]. The hybrid model is analogous to previous hybrid models [36]. The LME model is also a hybrid model integrating gain adaptive models, the fixed effects matrix, and stochastic processes; namely the random effects matrix. The LME model can only provide limited information regarding the biological mechanisms responsible for tinnitus perception. The random effects provide additional data to inform the existing biologically inspired fixed effects. Future work is required to extend the MPM to incorporate a biologically faithful approach to the relative perception of a sound replacing the need for subjective likeness data used in the LME model.

Further work is required to identify other characteristics of tinnitus such as the quality of a person's tinnitus, for example, a ringing or a hissing sound. The LME model is only applicable to continuous data and not categorical data such as the quality of a person's tinnitus.

\section{CONCLUSION}

The LME model provides the most accurate perceptual model of tinnitus to date as it shows the greatest correlation between the perceived tinnitus pitches and the modelled tinnitus pitches compared with all other perceptual models of tinnitus. The success of this model gave confidence to investigate other perceptual aspects of tinnitus, namely tinnitus loudness.

Most computational models of tinnitus replicate activity associated with tinnitus but only [36] has quantitatively explored the perception of sound emerging from abnormal tinnitus behaviour. This work aimed to extract perceptual aspects of tinnitus from the abnormal behaviour previously modelled and improve upon existing models of tinnitus perception by accounting for the heterogeneity in the tinnitus population.

The initial approach was to replicate existing methodologies for modelling tinnitus pitch using only modelled signals of the ascending auditory pathway [36]. The poor performance of the MPM and indeed the results of previous models showed that the bottom-up information does not contain everything needed to capture the perception of a sound. The individualism of sound perception motivated the investigation of LME models which capture random effects which were used to account for the subjective and individual nature of tinnitus.

As the top-down signals cannot be easily observed, subjective assessment of an individual's perception of sounds relative to their tinnitus was used as known random effects. As the sound stimuli were the same across all subjects this information provided an individual baseline for each person allowing the heterogeneity across subjects to be captured using a single model. Accurate models of tinnitus pitch and loudness perception were created by using subjective information from individuals on their perception of sounds similar to their tinnitus. These models could be used to assist with the assessment and validation of tinnitus pitch and loudness which is currently only obtainable through subjective measures. Additionally it could be used alongside longitudinal studies of tinnitus to track changes in a person's tinnitus; for example, a person's tinnitus before and after a treatment plan. This approach has provided a new state-of-the-art technique to perceptual modelling of tinnitus. 


\section{ACKNOWLEDGMENT}

The authors would like to thank Dr. Ovidiu König for providing Dataset 1 and Prof Larry Roberts and Dave Thompson for providing Dataset 2 from their previous studies. This work was completed under a PhD studentship supported by the Department of Education and Learning (DEL), Northern Ireland, U.K..

\section{REFERENCES}

[1] Bhatt, Jay M and Lin, Harrison W and Bhattacharyya, Neil. Prevalence, severity, exposures, and treatment patterns of tinnitus in the United States. JAMA Otolaryngology-Head \& Neck Surgery, 142(10):959-965, 2016.

[2] Madani, G and Connor, SEJ. Imaging in pulsatile tinnitus Clinical radiology, 64(3):319-328, 2009.

[3] Shore, Susan E and Roberts, Larry E and Langguth, Berthold. Maladaptive plasticity in tinnitus-triggers, mechanisms and treatment. Nature Reviews Neurology, 12(3):150, 2016.

[4] Henry, James A and Griest, Susan and Zaugg, Tara L and Thielman, Emily and Kaelin, Christine and Galvez, Gino and Carlson, Kathleen F. Tinnitus and hearing survey: a screening tool to differentiate bothersome tinnitus from hearing difficulties. American Journal of Audiology, 24(1):66-77, 2015.

[5] Wu, Calvin and Stefanescu, Roxana A and Martel, David T and Shore, Susan E. Tinnitus: maladaptive auditory-somatosensory plasticity. Hearing research, 334:20-29, 2016.

[6] Pawel J Jastreboff. Characteristics of tinnitus and etiology of associated hearing loss: a study of 123 patients. International Tinnitus Journal, 8(1):37-44, 2002.

[7] Cécile Nicolas-Puel, Ruth Lloyd Faulconbridge, Mattieu Guitton, JeanLuc Puel, Michel Mondain, and Alain Uziel. Characteristics of tinnitus and etiology of associated hearing loss: a study of 123 patients International Tinnitus Journal, 8(1):37-44, 2002.

[8] Hesse, Lara Li and Bakay, Warren and Ong, Hui-Ching and Anderson, Lucy and Ashmore, Jonathan and McAlpine, David and Linden, Jennifer and Schaette, Roland. Non-monotonic relation between noise exposure severity and neuronal hyperactivity in the auditory midbrain. Frontiers in neurology, 7:133, 2016.

[9] Roland Schaette and David McAlpine. Tinnitus with a normal audiogram: physiological evidence for hidden hearing loss and computational model. The Journal of Neuroscience, 31(38):13452-13457, 2011.

[10] Viana, Lucas $M$ and O'Malley, Jennifer T and Burgess, Barbara J and Jones, Dianne D and Oliveira, Carlos ACP and Santos, Felipe and Merchant, Saumil N and Liberman, Leslie D and Liberman, M Charles. Cochlear neuropathy in human presbycusis: Confocal analysis of hidden hearing loss in post-mortem tissue. Hearing research, 327:77-88, 2015.

[11] Gault Richard, McGinnity Thomas Martin and Coleman Sonya. Computational model of thalamocortical dysrhythmia in tinnitus sufferers IEEE Transactions on Neural Systems and Rehabilitation Engineering, 26(9):1845-1857,2018, doi: 10.1109/TNSRE.2018.2863740.

[12] JS Zhang, JA Kaltenbach, DA Godfrey, and J Wang. Origin of hyperactivity in the hamster dorsal cochlear nucleus following intense sound exposure. Journal of Neuroscience Research, 84(4):819-831, 2006.

[13] TJ Brozoski, CA Bauer, and DM Caspary. Elevated fusiform cell activity in the dorsal cochlear nucleus of chinchillas with psychophysical evidence of tinnitus. Journal of Neuroscience, 22(6):2383-2390, 2002

[14] Henry E Heffner and Ian A Harrington. Tinnitus in hamsters following exposure to intense sound. Hearing Research, 170(1-2):83-95, 2002.

[15] James A Kaltenbach, Mark A Zacharek, Jinsheng Zhang, and Sarah Frederick. Activity in the dorsal cochlear nucleus of hamsters previously tested for tinnitus following intense tone exposure. Neuroscience Letters, 355(1-2):121-125, 2004.

[16] James A Kaltenbach and Jinsheng Zhang. Intense sound-induced plasticity in the dorsal cochlear nucleus of rats: evidence for cholinergic receptor upregulation. Hearing Research, 226(1-2):232-243, 2007.

[17] James A Kaltenbach. The dorsal cochlear nucleus as a participant in the auditory, attentional and emotional components of tinnitus. Hearing Research, 216:224-234, 2006.

[18] Kaltenbach, James A and Godfrey, Donald A and Neumann, John B and McCaslin, Devin L and Afman, Chad E and Zhang, Jinsheng. Changes in spontaneous neural activity in the dorsal cochlear nucleus following exposure to intense sound: relation to threshold shift. Hearing research, 124(1-2):78-84, 1998.
[19] Kaltenbach, James A and Afman, Chad E. Hyperactivity in the dorsal cochlear nucleus after intense sound exposure and its resemblance to toneevoked activity: a physiological model for tinnitus. Hearing research, 140(1-2):165-172, 2000.

[20] Kaltenbach, James A and Rachel, John D and Mathog, T Alecia and Zhang, Jinsheng and Falzarano, Pamela R and Lewandowski, Matthew. Cisplatin-induced hyperactivity in the dorsal cochlear nucleus and its relation to outer hair cell loss: relevance to tinnitus. American Physiological Society Bethesda, MD, 88(2):699-714, 2002.

[21] Jianwen Wendy Gu, Barbara S Herrmann, Robert A Levine, and Jennifer R Melcher. Brainstem auditory evoked potentials suggest a role for the ventral cochlear nucleus in tinnitus. Journal of the Association for Research in Otolaryngology, 13(6):819-833, 2012.

[22] NF Manzoor, Y Gao, F Licari, and JA Kaltenbach. Comparison and contrast of noise-induced hyperactivity in the dorsal cochlear nucleus and inferior colliculus. Hearing Research, 295:114-123, 2013.

[23] NF Manzoor, Frank G Licari, Michael Klapchar, RL Elkin, Yuan Gao, G Chen, and JA Kaltenbach. Noise-induced hyperactivity in the inferior colliculus: its relationship with hyperactivity in the dorsal cochlear nucleus. Journal of Neurophysiology, 108(4):976-988, 2012.

[24] Gregory J Basura, Seth D Koehler, and Susan E Shore. Bimodal stimulus timing-dependent plasticity in primary auditory cortex is altered after noise exposure with and without tinnitus. Journal of Neurophysiology, 114(6):3064-3075, 2015.

[25] Sönke Ahlf, Konstantin Tziridis, Sabine Korn, Ilona Strohmeyer and Holger Schulze. Predisposition for and prevention of subjective tinnitus development. PLoS One, 7(10):e44519, 2012.

[26] Weisz, Nathan and Müller, Simona and Schlee, Winfried and Dohrmann, Katalin and Hartmann, Thomas and Elbert, Thomas. The neural code of auditory phantom perception. Journal of Neuroscience, 27(6):1479-1484, 2007.

[27] Ana Belén Elgoyhen, Berthold Langguth, Dirk De Ridder, and Sven Vanneste. Tinnitus: perspectives from human neuroimaging. Nature Reviews Neuroscience, 16(10):632, 2015.

[28] Peyman Adjamian, Magdalena Sereda, and Deborah A Hall. The mechanisms of tinnitus: perspectives from human functional neuroimaging. Hearing Research, 253(1-2):15-31, 2009.

[29] Vanneste, Sven and To, Wing Ting and De Ridder, Dirk. Tinnitus and neuropathic pain share a common neural substrate in the form of specific brain connectivity and microstate profiles. Progress in NeuroPsychopharmacology and Biological Psychiatry, 88:388-400, 2019.

[30] Cederroth, Christopher R and Gallus, Silvano and Hall, Deborah A and Kleinjung, Tobias and Langguth, Berthold and Maruotti, Antonello and Meyer, Martin and Norena, Arnaud and Probst, Thomas and Pryss, Rüdiger Christoph and others. Towards an Understanding of Tinnitus Heterogeneity. Frontiers in aging neuroscience, 11:53, 2019.

[31] Henry, James A and Roberts, Larry E and Caspary, Donald M and Theodoroff, Sarah M and Salvi, Richard J. Underlying mechanisms of tinnitus: review and clinical implications. Journal of the American Academy of Audiology, 25(1):5-22, 2014.

[32] Lucas C Parra and Barak A Pearlmutter. Illusory percepts from auditory adaptation. The Journal of the Acoustical Society of America, 121(3):1632-1641, 2007.

[33] Ian C Bruce, Harjeet S Bajaj, and Jennifer Ko. Lateral-inhibitorynetwork models of tinnitus. In In Proceedings of the 5th IFAC Symposium on Modelling and Control in Biomedical Systems. Citeseer, 2003.

[34] Roland Schaette and Richard Kempter. Development of hyperactivity after hearing loss in a computational model of the dorsal cochlear nucleus depends on neuron response type. Hearing Research, 240(1):57-72, 2008.

[35] Roland Schaette and Richard Kempter. Development of tinnitus-related neuronal hyperactivity through homeostatic plasticity after hearing loss: a computational model. European Journal of Neuroscience, 23(11):31243138, 2006.

[36] Roland Schaette and Richard Kempter. Predicting tinnitus pitch from patients' audiograms with a computational model for the development of neuronal hyperactivity. Journal of Neurophysiology, 101(6):3042-3052, 2009.

[37] Krauss, Patrick and Tziridis, Konstantin and Metzner, Claus and Schilling, Achim and Hoppe, Ulrich and Schulze, Holger. Stochastic resonance controlled upregulation of internal noise after hearing loss as a putative cause of tinnitus-related neuronal hyperactivity. Frontiers in Neuroscience, 10:597, 2016.

[38] Noda, Kazuhiro and Kitahara, Tadashi and others. Sound Change Integration Error: An Explanatory Model of Tinnitus. Frontiers in Neuroscience, 12:831, 2018.

[39] Sametsky, E. A., Turner, J. G., Larsen, D., Ling, L., and Caspary, D. M. (2015). Enhanced GABAA-mediated tonic inhibition in auditory thalamus 
of rats with behavioral evidence of tinnitus. Journal of Neuroscience, 35(25):9369-9380.

[40] Larry E Roberts, Graeme Moffat, Michael Baumann, Lawrence M Ward, and Daniel J Bosnyak. Residual inhibition functions overlap tinnitus spectra and the region of auditory threshold shift. Journal of the Association for Research in Otolaryngology, 9(4):417-435, 2008.

[41] Ovidiu König, Roland Schaette, Richard Kempter, and Manfred Gross. Course of hearing loss and occurrence of tinnitus. Hearing Research, 221(1):59-64, 2006.

[42] Muhammad SA Zilany, Ian C Bruce, and Laurel H Carney. Updated parameters and expanded simulation options for a model of the auditory periphery. The Journal of the Acoustical Society of America, 135(1):283286, 2014.

[43] Brian CJ Moore and Brian R Glasberg. Suggested formulae for calculating auditory-filter bandwidths and excitation patterns. The Journal of the Acoustical Society of America, 74(3):750-753, 1983.

[44] https://www.urmc.rochester.edu/labs/carney/publications-code/auditorymodels.aspx.

[45] Ian C bruce and Muhammad SA Zilany. AModelling the effects of cochlear impairment on the neural representation of speech in the auditory nerve and primary auditory cortex. Proceedings of the International Symposium on Auditory and Audiological Research, 1:1-10, 2007.

[46] Donald D Greenwood. A cochlear frequency-position function for several species-29 years later. The Journal of the Acoustical Society of America, 87(6):2592-2605, 1990.

[47] M Charles Liberman. Auditory-nerve response from cats raised in a low-noise chamber. The Journal of the Acoustical Society of America, 63(2):442-455, 1978.

[48] Heil, Peter and Peterson, Adam J. Basic response properties of auditory nerve fibers: a review. Cell and tissue research, 361(1):129-158, 2015.

[49] Ohlemiller, KK and Echteler, SM. Functional correlates of characteristic frequency in single cochlear nerve fibers of the Mongolian gerbil. Journal of Comparative Physiology A, 167(3):329-338, 1990.

[50] Kenneth E Hancock and Herbert F Voigt. Wideband inhibition of dorsal cochlear nucleus type iv units in cat: a computational model. Annals of Biomedical Engineering, 27(1):73-87, 1999.

[51] George M Gerken. Central tinnitus and lateral inhibition: an auditory brainstem model. Hearing Research, 97(1):75-83, 1996.

[52] G Langner and E Wallhäusser-Franke. Computer simulation of a tinnitus model based on labelling of tinnitus activity in the auditory cortex. In Proceedings of the Sixth International Tinnitus Seminar, pages 20-25, 1999.

[53] A Kral and V Majernik. On lateral inhibition in the auditory system. General Physiology and Biophysics, 15:109-128, 1996.

[54] Diehl, P. U. and Schaette, R. (2015). Abnormal auditory gain in hyperacusis: investigation with a computational model. Frontiers in Neurology, 6. 